\title{
Soil microbial responses to biochars varying in particle size, surface and pore properties
}

\begin{abstract}
Biochars are known for their heterogeneity, especially in pore and surface structure associated with pyrolysis processes and sources of feedstocks. The surface area of biochar is likely to be an important determinant of the extent of soil microbial attachment, whereas the porous structure of biochar is expected to provide protection for soil microorganisms. Potential interactions between biochars from different sources and with different particle sizes were investigated in relation to soil microbial properties in a short-term incubation study. Three particle size (sieved) fractions (0.5-1.0, 1.0-2.0 and 2.0-4.0 mm) from three woody biochars produced from jarrah wood, jarrah and wandoo wood and Australian wattle branches, respectively, were incubated in soil at $25{ }^{\circ} \mathrm{C}$ for $56 \mathrm{~d}$. Observation by scanning electron microscopy (SEM) and characterisation of pore and surface area showed that all three woody biochars provided potential habitats for soil microorganisms due to their high porosity and surface areas. The biochars were structurally heterogeneous, varying in porosity and surface structure both within and between the biochar sources. After the 56-d incubation, hyphal colonisation was observed on biochar surfaces and in larger biochar pores. Soil clumping occurred on biochar particles, cementing and covering exposed biochar pores. This may have altered surface area and pore availability for microbial colonisation. Transient changes in soil microbial biomass, without a consistent trend, were observed among biochars during the 56-d incubation.
\end{abstract}

Keyword: Microbial biomass; Microbial colonisation; Microbial habitats; Porosity; Scanning electron microscopy; Surface structure 